\title{
Is Purkinje neuron hyperpolarisation important for cerebellar synaptic plasticity? A retrospective and prospective analysis
}

Abbreviated title: Purkinje neuron hyperpolarisation and plasticity

$$
\text { Marco Canepari }{ }^{1,2,3,{ }^{*}}
$$

${ }^{1}$ Univ. Grenoble Alpes, CNRS, LIPhy, F-38000 Grenoble, France. ${ }^{2}$ Laboratories of Excellence, Ion Channel Science and Therapeutics, France. ${ }^{3}$ Institut National de la Santé et Recherche Médicale, France.

Lead contact and correspondance: marco.canepari@univ-grenoble-alpes.fr

Type of article: Review.

Number of pages: 20. Number of figures: 4 .

Number of characters in the title: 106. Number of words for abstract: 180. Number of references: 102

Conflict of interests: None

Acknowledgements: This work was supported by the Agence Nationale de la Recherche through the Labex Ion Channels Science and Therapeutics: program number ANR-11-LABX-0015.

Author contributions: MC wrote the paper. 


\section{Abstract}

Two recent studies have demonstrated that the dendritic $\mathrm{Ca}^{2+}$ signal associated with a climbing fibre (CF) input to the cerebellar Purkinje neuron $(P N)$ depends on the membrane potential $\left(V_{m}\right)$. Specifically, when the cell is hyperpolarised, this signal is mediated by T-type voltage-gated $\mathrm{Ca}^{2+}$ channels; in contrast, when the cell is firing, the CF-PN signal is mediated by $\mathrm{P} / \mathrm{Q}$-type voltage-gated $\mathrm{Ca}^{2+}$ channels. When the $\mathrm{CF}$ input is paired with parallel fibre (PF) activity, the signal is locally amplified at the sites of PF-activated synapses according to the $V_{m}$ at the time of the CF input, suggesting that the standing $V_{m}$ is a critical parameter for the induction of PF synaptic plasticity. In this review I analyse how the $V_{m}$ can potentially play a role in cerebellar learning focussing, in particular, on the hyperpolarised state that appears to occur episodically, since PNs are mostly firing under physiological conditions. By revisiting the recent literature reporting in vivo recordings and synaptic plasticity studies, I speculate on how a putative role of the $P N V_{m}$ can provide an interpretation for the results of these studies.

Keywords: Purkinje neuron, membrane potential, calcium channels, synaptic plasticity, Climbing fibre, Parallel fibres. 


\section{Introduction}

In the vertebrate nervous system, the cerebellum integrates the incoming motor and sensory information to produce a feedback output from the cerebellar nuclei to the motor system that controls movements [1]. This output is precisely shaped by the inhibitory input to cerebellar nuclei neurons from cerebellar Purkinje neurons (PNs) in the cerebellar cortex, which also processes incoming motor information. Specifically, PNs fire action potentials at variable frequencies and these frequencies are modulated by the motor information received from the mossy fibres and processed by cerebellar granule cells (CGCs) that send excitatory parallel fibre (PF) inputs to PNs. The PN firing frequency can either increase or decrease during motor activity according to the balance of excitation versus feed-forward inhibition by molecular layer interneurons (MLIs) also excited by the same PF inputs [2]. But the whole cerebellar system also receives a large sensory excitatory input through the climbing fibres (CFs) from the brainstem inferior olive targeting both PNs and cerebellar nuclei neurons. According to the early theory proposed by Marr [3] and Albus [4], the principal role of the CF input is to provide an "error" signal that weakens the concomitantly active PFs input, in this way permitting a continuous learning of fine motor coordination. This theory was finally validated by the discovery that pairing PF and CF activity can induce long-term depression (LTD) of activated PF synapses [5]. Since then, it became evident that physiological PF synaptic depression is complex and highly heterogeneous in the way it is triggered throughout the cerebellar cortex [6], and that PF plasticity must be bidirectional with synaptic weakening compensated by counterbalancing mechanisms of long-term potentiation (LTP) $[7,8]$.

All CF-mediated forms of homosynaptic plasticity in PNs, although different, require the ability of the CF input to target a triggering signal exclusively to the concomitantly activated PF synapses. This signal that coincidently detects the occurrence of PF and CF inputs and that can initiate a biochemical process leading to a change in the postsynaptic strength is a transient elevation of intracellular $\mathrm{Ca}^{2+}$ concentration [9]. More precisely, when the occurrence of a CF excitatory postsynaptic potential (EPSP) is preceded by PF activation, the $\mathrm{Ca}^{2+}$ transient associated with the CF-EPSP, at the location of PF synapses only, is larger than the $\mathrm{Ca}^{2+}$ transient associated with the CF-EPSP without PF activation (unpaired CF-EPSP), and it is referred as "supralinear" $\mathrm{Ca}^{2+}$ signal $[10,11]$. Whereas CGCs form a complex circuit of $\sim 175,000$ contacts in the dendritic spines of PN dendrites [12], the CF input is a unique synapse with over 500 realising sites [13] targeting the soma and the initial dendritic segment and generating a large 
depolarisation throughout the dendritic arborisation leading to a widespread $\mathrm{Ca}^{2+}$ transient [14-17]. The mechanisms permitting the CF-EPSP to mediate localised $\mathrm{Ca}^{2+}$ signals are therefore fundamental for PF synaptic plasticity. A supralinear $\mathrm{Ca}^{2+}$ signal, i.e. a larger increase in intracellular $\mathrm{Ca}^{2+}$ concentration can be generated by a larger flux of $\mathrm{Ca}^{2+}$ into the cytosol, but also by an increase of free $\mathrm{Ca}^{2+}$ due to a transient saturation of the endogenous $\mathrm{Ca}^{2+}$ buffer (ECB). PN dendrites have an exceptionally high $\mathrm{ECB}$ [18] formed mainly by two mobile high-affinity $\mathrm{Ca}^{2+}$ binding proteins: Calbindin-D28k and Parvalbumin $[19,20]$. It has been demonstrated that PN-ECB can be saturated by $\mathrm{Ca}^{2+}$ photolysis [21] or by fast $\mathrm{Ca}^{2+}$ influx mediated by the PF local depolarisation [16]. More interestingly, it was recently shown that the slow $\mathrm{Ca}^{2+}$ influx induced by PF-activated type-1 metabotropic glutamate receptors (mGluR1s) can transiently saturate the ECB [11]. Thus, PF inputs through mGluR1 activation can determine the condition to target a CF-mediated $\mathrm{Ca}^{2+}$ signal to the synaptic sites where synaptic plasticity has to occur by locally amplifying the $\mathrm{Ca}^{2+}$ transient associated with the CF-EPSP. The source of this $\mathrm{Ca}^{2+}$ transient, however, is not the same at any membrane potential $\left(\mathrm{V}_{\mathrm{m}}\right)$ [17]. When the $\mathrm{PN}$ is at a quiescent hyperpolarising state, the CFmediated depolarisation transient activates T-type voltage-gated $\mathrm{Ca}^{2+}$ channels (VGCCs) and A-type voltage-gated $\mathrm{K}^{+}$channels that limit the activation of P/Q-type VGCCs preventing dendritic $\mathrm{Ca}^{2+}$ spikes. When instead the PN is at a more depolarised firing state, both $\mathrm{T}$ and $\mathrm{A}$ channels inactivate and the CFmediated depolarisation activates P/Q-type VGCCs enabling $\mathrm{Ca}^{2+}$ spikes. Notably, both $\mathrm{Ca}^{2+}$ sources are potentiated by PF-mediated mGluR1 activation, but not in the same way. $\mathrm{Ca}^{2+}$ spikes via $\mathrm{P} / \mathrm{Q}$ channels are boosted by mGluR1-mediated inactivation of A channels [22] whereas T-channels are directly potentiated by mGluR1s at hyperpolarised states [23,24]. Notably, the potentiation of T-type VGCCs is mediated by the same mGluR1 pathway responsible for the slow mGluR1-mediated $\mathrm{Ca}^{2+}$ influx [25] suggesting a possible co-localisation of the two processes. Thus, beside the voltage independent amplification through local saturation of the ECB, the supralinear $\mathrm{Ca}^{2+}$ signal associated with a CF-EPSP includes an increase in $\mathrm{Ca}^{2+}$ influx through the plasma membrane and has a different origin depending on whether the initial $V_{m}$ is at a hyperpolarised or at a firing state. This fact suggests that the standing $V_{m}$ of PNs plays a pivotal role in synaptic plasticity and cerebellar learning.

This review is a retrospective and prospective analysis of the possible role of $P N V_{m}$ in synaptic plasticity. In particular, I focus on the putative role of PN hyperpolarisation since this state is likely episodic while the supralinear $\mathrm{Ca}^{2+}$ signal appears to specifically target activated PF synapses. In the following 
sections, I first review in detail the biophysical origin of mGluR1-dependent supralinear $\mathrm{Ca}^{2+}$ signals. Then, I review some of the most important findings on in vivo activity and provide interpretations in light of a possible role of the $\mathrm{PN} \mathrm{V}_{\mathrm{m}}$, I analyse the possible activation of $\mathrm{K}^{+}$channels that may generate episodes of hyperpolarisation and I speculate on how episodic hyperpolarisation can relate to the rules that govern synaptic plasticity. Finally, I propose a perspective of future research directions to tackle the different hypotheses analysed in this review.

\section{Overview of the origin of mGluR1 supralinear $\mathrm{Ca}^{2+}$ signals in Purkinje neurons}

I start this analysis with an overview of the recent findings that motivated this review. The first findings concern the biophysical mechanisms governing the dendritic $\mathrm{V}_{\mathrm{m}}$ and $\mathrm{Ca}^{2+}$ transients associated with the CF-EPSP at different initial $\mathrm{V}_{\mathrm{m}}$ [17]. Modifying a previous model [26] to match combined $\mathrm{V}_{\mathrm{m}}$ and $\mathrm{Ca}^{2+}$ imaging data, the response of a dendritic compartment to a CF depolarising transient was explained in detail with the synergistic activation of six channels. These channels are divided in two different sets that are selectively activated at different initial $V_{m}$. By focussing on the two types of VGCCs and on the A-type $\mathrm{K}^{+}$channel (Fig. 1a), when the dendrite is hyperpolarised a CF-EPSP activates T-type VGCCs and A-type $\mathrm{K}^{+}$channels channels that limit the dendritic depolarising transient below $\sim 10 \mathrm{mV}$, preventing the activation of $\mathrm{P} / \mathrm{Q}$-type VGCCs. When in contrast the dendrite is depolarised, $\mathrm{T}$ and $\mathrm{A}$ channels are inactivated and the CF-EPSP can eventually activate $P / Q$ channels eliciting $\mathrm{Ca}^{2+}$ spikes. A second study [11] has unravelled the mechanisms that allow the $\mathrm{Ca}^{2+}$ transient associated with a CF-EPSP, following PF activity, to be amplified locally at the sites of pre-activated PF synapses (Fig. 1b). A first mechanism, independent of the initial $\mathrm{V}_{\mathrm{m}}$, is determined by the $\mathrm{Ca}^{2+}$ influx through an mGluR1-activated non-selective cation conductance $[27,28]$. This channel is believed to be the C3-type transient receptor potential (TRPC3) [29], but it was also shown that mGluR1s can activate GluD2 delta "orphan" glutamate receptors [30]. During the precise time window of mGluR1 action following PF bursting activity, $\mathrm{Ca}^{2+}$ entering the cell through this channel binds to ECBs locally lowering the buffer capacity of the cell. Since this is produced by synaptically activated receptors, it can be segregated to activated synapses, i.e. it can co-localise with activated spines [31] depending on their geometry [32]. The mechanism of local ECB saturation amplifies both the $\mathrm{Ca}^{2+}$ signal mediated by T-type VGCCs at hyperpolarised states and the $\mathrm{Ca}^{2+}$ signal mediated by P/Q-type VGCCs at depolarised states combining, in the two cases, with two distinct further mechanisms 
contributing to the supralinear $\mathrm{Ca}^{2+}$ signals. The mechanism of boosting $\mathrm{P} / \mathrm{Q}$ channel $\mathrm{Ca}^{2+}$ influx, due to the larger mGluR1-dependent inactivation of A channels [22] is not local. In contrast, the mechanism of boosting $\mathrm{T}$ channel $\mathrm{Ca}^{2+}$ influx, due to the direct mGluR1-dependent potentiation of these channels [23], is likely occurring exclusively at activated PF synapses. Altogether, these findings lead to the question on whether the $V_{m}$ is actually a key parameter in the induction of synaptic plasticity and, in particular, whether the apparently rare states of $V_{m}$ hyperpolarisation are fundamental episodes necessary for cerebellar learning. In the next section I review the state-of-the art literature on the $\mathrm{V}_{\mathrm{m}}$ in vivo in light of the possible occurrence of hyperpolarised states.

\section{Evidence of physiological hyperpolarisation of Purkinje neurons in vivo}

Direct measurement of $\mathrm{V}_{\mathrm{m}}$ by in vivo patch clamp recordings in PNs were obtained both from anesthetised [33-35] and awake animals [2]. In anesthetised rodents, independently of the anaesthesia procedure, $\mathrm{PN} \mathrm{V}_{\mathrm{m}}$ is characterised by bistability consisting on the ability of alternating between hyperpolarised quiescent states and depolarised firing states associated with simple spikes [33], similarly to what was reported in vitro [36]. While PN bistability is due to intrinsic membrane properties (see for example [37]), a CF input can switch the $\mathrm{V}_{\mathrm{m}}$ from one state to the other [33]. Notably, the $\mathrm{Ca}^{2+}$ transient associated with a CF-EPSP depends on the PN state [34], in the same way as it depends on the initial $V_{m}$ in brain slices [17]. Yet, the ability of PNs to switch between two states, supposedly associated with the possibility to activate either T-type or P/Q-type VGCCs, does not answer the question on how frequent the hyperpolarised state occurs. Less invasive in vivo extracellular recordings have shown that the frequency of simple spike firing is on average higher in awake mice with respect to anesthetised mice, ranging from $\sim 30 \mathrm{~Hz}$ to $\sim 100 \mathrm{~Hz}$ [38]. The variability in spiking frequency reflects the cerebellar modules where modules expressing zebrin exhibit lower frequencies [39] and this specificity is mediated by TRPC3 channels [40]. In all cases, these relatively high spiking frequencies indicate that hyperpolarising states lasting more than 100 ms must be, under physiological conditions, only episodic. This first conclusion leads to the second important question, i.e. how synaptic activity may lead to these episodic hyperpolarised states. While a CF input alone can theoretically switch from one PN state to another, the occurrence of complex spikes in vivo, indicative of a CF input, does not silence simple spiking activity (see for example [38]) since the transient hyperpolarisation mediated by $\mathrm{K}^{+}$channels activation, in particular 
$\mathrm{Ca}^{2+}$-activated BK channels $[41,17]$ is lasting only a few milliseconds. Thus, episodes of sustained hyperpolarisation can be only induced either by PF activity alone or by concomitant PF activity and CF inputs. A possibility is that hyperpolarisation episodes have a network origin being caused by outward $\mathrm{Cl}^{-}$ currents through $\mathrm{GABA}_{\mathrm{A}}$ receptors activated by cerebellar interneurons. PF axons projecting onto PNs also excite MLIs producing feed-forward inhibition to the same PNs and this mechanism regulates behaviours associated with synaptic plasticity and spiking activity in PNs [42,43]. Furthermore, the contribution of feed-forward inhibition in determining the firing rate is enhanced during self-paced locomotion where some PNs increase their spiking frequency whereas others decrease it [2]. This finding leads to a straightforward hypothesis illustrated in Fig. 2. In PNs where the firing frequency increases, and therefore the standing $\mathrm{V}_{\mathrm{m}}$ becomes more depolarised, a CF-EPSP would likely activate P/Q-type VGCCs whereas in PNs where the firing frequency decreases, and the standing $V_{m}$ becomes more hyperpolarised, a CF-EPSP would more likely activate T-type VGCCs. But how MLI inputs to PNs affect the CF response and synaptic plasticity? These questions were investigated by Rowan et al. [44] who showed that concomitant optogenetic stimulation of MLIs reduced the CF-mediated $\mathrm{Ca}^{2+}$ transient and when this protocol was paired with PF stimulation it changed the polarity of PF long-term synaptic plasticity from depression to potentiation (i.e. from LTD to LTP). In addition, while CF stimulation combined with head rotation produced an adaptive increase in the vestibulo-ocular reflex, concomitant optogenetic stimulation of MLIs reversed the effect into an adaptive decrease. The straightforward possible interpretation according to the scheme of Fig. 2 would be that MLIs drive the PN to a hyperpolarised state changing the polarity of PF synaptic plasticity. However, activation of molecular layer inhibitory synapses does not only hyperpolarise the $\mathrm{V}_{\mathrm{m}}$, but also increases the dendritic membrane conductance decreasing the propagation of the CF depolarising transient throughout the dendrites. Hence, while molecular layer "disinhinibition" increases the CF-mediated $\mathrm{Ca}^{2+}$ transient during behaviourally-induced PF activity, MLI activation suppresses local supralinear $\mathrm{Ca}^{2+}$ signals [45], a result in striking contrast with the large supralinear $\mathrm{Ca}^{2+}$ signal observed with hyperpolarised states obtained by somatic current injection [11]. Thus, while MLIs may in principle allow, in a portion of PNs, the activation of T-type VGCCs, the results obtained in these studies can be alternatively attributed to a shunting inhibition of the CF depolarising wave. The specific investigation of the occurrence of hyperpolarising episodes in vivo is only at a preliminary stage. It must be taken into account that T-type VGCCs recover from inactivation in a few milliseconds [46] and, therefore, an hyperpolarisation episode synchronising with a CF-EPSP can be as 
short as 100 ms to allow a local supralinear $\mathrm{Ca}^{2+}$ signal mediated by these channels. For example, within feed-forward inhibition, a spike from one single MLI can alone transiently inhibit PN firing [35], but MLIs can be also excited by glutamate spillover from a CF input [47]. The hypothesis that episodes of PN hyperpolarisation playing a role in synaptic plasticity are determined by activation of $\mathrm{Cl}^{-}$implies that the underlying mechanisms are mainly governed by the cerebellar network. Yet, an alternative hypothesis is that hyperpolarisation episodes are determined by the history of the PN signals and governed by intrinsic mechanisms leading to activation of $\mathrm{K}^{+}$currents. This hypothesis is analysed in the next section.

\section{Mechanisms of episodic hyperpolarisation of Purkinje neurons mediated by $\mathrm{K}^{+}$channels}

A prolonged activation of $\mathrm{K}^{+}$conductance can drive $\mathrm{PN} \mathrm{V}_{\mathrm{m}}$ towards the reversal potential of $\mathrm{K}^{+}$. This can be produced by a large $\mathrm{Ca}^{2+}$ transient activating $\mathrm{Ca}^{2+}$-gated $\mathrm{K}^{+}$channels or by $\mathrm{K}^{+}$channels activated by other mechanisms. Among the different types of $\mathrm{Ca}^{2+}$-gated $\mathrm{K}^{+}$channels, the "small-conductance" $\mathrm{K}^{+}$ (SK) channel is reported to play a role in different cellular processes in PNs, including in the calcium transients in dendritic spines [48]. Although the expression of this channel decreases with development [49], it was recently reported that SK channels are highly expressed in dendritic spines co-localising with mGluR1s [50] and that they are down-regulated during cerebellar learning increasing PN excitability [51]. In general, SK channels can provide a medium-slow afterhyperpolarisation after PF bursting. The other type of $\mathrm{Ca}^{2+}$-gated $\mathrm{K}^{+}$channel expressed in $\mathrm{PNs}$ is the "big-conductance" $\mathrm{K}^{+}(\mathrm{BK})$ channel that co-localise with P/Q-type VGCCs [52] and is activated by dendritic $\mathrm{Ca}^{2+}$ spikes $[41,17]$. But BK channels are also activated by $\mathrm{Ca}^{2+}$ influx via inositol 1,4,5-triphosphate (InsP3) receptors activated by mGluR1s with a faster kinetics with respect to the slow $\mathrm{Ca}^{2+}$ influx [53]. Notably, since InsP3 receptors are highly expressed in PN dendrites [54], several studies have linked PF activation of mGluR1s to $\mathrm{Ca}^{2+}$ release from internal stores via these receptors $[55,56]$ leading to the hypothesis that this signal is the trigger for PF-LTD [57,58]. While $\mathrm{Ca}^{2+}$ release from internal stores does not contribute to the CF dependent supralinear $\mathrm{Ca}^{2+}$ signal [11], InsP3 receptor activation requires dendritic depolarisation [53] raising the hypothesis that this event may play a physiological role in generating hyperpolarisation short episodes. Finally, $\mathrm{Ca}^{2+}$ release from stores activating $\mathrm{Ca}^{2+}$-gated $\mathrm{K}^{+}$channels can also occur via ryanodine receptors following an intense stimulation [59], a mechanism that was also associated with the induction of plasticity [60]. Altogether, the ensemble of possible mechanisms leading to activation of $\mathrm{Ca}^{2+}$-gated $\mathrm{K}^{+}$channels is 
summarised in Fig. 3a. In addition to the pathways analysed above, $\mathrm{K}^{+}$channels can be activated by other synaptic mechanisms. PF activity activates type-7 metabotropic glutamate receptors (mGluR7) and this mechanism can transiently silence PN activity after cerebellar learning [61]. Specifically, mGluR7s couple with Kir3/GIRK K+ channels [62] that are expressed in PNs [63]. The same channels are activated by inhibitory synapses by coupling with metabotropic $\mathrm{GABA}_{B}$ receptors [64]. Altogether, the two mechanisms leading to activation of Kir3/GIRK channels are summarised in Fig. 3b. In light of this general analysis on what can produce hyperpolarisation transients during PF or CF activity, it is crucial to analyse, next, how these mechanisms may relate to the different protocols leading to synaptic plasticity. This issue is addressed in the next section.

\section{How hyperpolarisation can relate to the induction of synaptic plasticity}

In the classical theory, the CF input combined with PF activity induces PF-LTD, a prediction that found numerous experimental conformations in brain slices experiments (see for example [9,65-67]). In the opposite direction, repetitive PF activity has been associated with induction of PF-LTP (see for example $[16,68,69])$, although strong PF stimulation can alone induce PF-LTD [70]. These findings led to the initial hypothesis that the amplitude of $\mathrm{a} \mathrm{Ca}^{2+}$ transient determines the direction of the PF long-term synaptic plasticity according to an "inverse BMC rule" [7] with respect to the equivalent BMC rule believed to govern cortical synaptic plasticity [71]. Yet, later, it became clear that the induction of PF synaptic plasticity is more complex [8] depending on many parameters. For instance, mGluR1-dependent PF-LTD [72-74] is also critically dependent on the geometry of activated PF fibres [75] since mGluR1s, located at the periphery of synaptic spines [76], are activated only by glutamate spillover by adjacent terminals [77] pointing out a potential importance of the arrangement of activated PFs forming "beams" capable of activating spines in close proximity [78]. The current opinion, however, is now questioning whether general rules of synaptic plasticity induction physiologically apply. First, PF-LTD is not always necessary for vestibular-ocular reflex adaptation $[79,80]$, being replaced by a rebound potentiation of inhibitory synapses to PNs [81]. Conversely, tactile stimulation in vivo can evoke a long-lasting increase in PN firing while synergistically reducing the MLI firing thanks to multiple plasticity mechanisms occurring at different cellular levels [82]. Second, PF-LTP can be expressed either at presynaptic terminals [83,84] or at postsynaptic spines [16,69], and the different forms of PF long-term synaptic plasticity, including PF-LTD, 
involve presynaptic mechanisms such as NMDA receptor activation $[85,86]$ triggering release of nitric oxide [87] and endocannabinoid receptor activation [88]. Yet, these presynaptic mechanisms are regulated by retrograde postsynaptic mechanisms [89-91], indicating that the synergy between presynaptic activity, controlled only by the principal motor input, and postsynaptic signalling, controlled also by the CF input, is a crucial determinant of synaptic plasticity. Finally, it was found that the rules for induction of PF plasticity are different in the flocculus and in the vermis [92], in the first case being narrowly tuned for a precise interval associated with oculomotor learning, while in the second case being highly variable possibly matching single cell functions. Thus, within the actual complicated scenarios of multiple rules of synaptic plasticity induction [93], the ability of $V_{m}$ to rapidly switch between two states characterised by different $\mathrm{Ca}^{2+}$ sources and pathways may be a critical factor and it may allow the CF input to drive synaptic plasticity in different directions [94].

Further theoretical studies on cerebellar plasticity have challenged the concept of "motor error", since the CF input carries a sensory and not a motor information [95]. Since a "sensory error" must be fully uncorrelated from the motor information in order to instruct the motor system in an independent way, the classical learning rule based on simple concomitant occurrence of PF and CF inputs has been replaced by a decorrelation learning rule based on positive or negative correlation of PF and CF activities [96]. This alternative learning theory brings up an intriguing question: can the $\mathrm{PN} \mathrm{V}_{\mathrm{m}}$ detect the type of correlation? A recent theoretical and experimental analysis has suggested that correlation detection is possible with two consecutive CF inputs separated by $100 \mathrm{~ms}$ [97]. In addition, in a more recent study, PF-LTD induction required the occurrence of two consecutive CF inputs at similar intervals [98]. Under certain circumstances, it is plausible that the first CF input during or just after PF activity can switch the standing $\mathrm{V}_{\mathrm{m}}$ from a depolarised state to an hyperpolarised state, priming the dendrite for the second CF input according to a scheme shown in Fig. 4a. The biophysical mechanism underlying this scenario can be the activation of $\mathrm{Ca}^{2+}$-activated $\mathrm{K}^{+}$channels by the first paired $\mathrm{CF}$ input that activates $\mathrm{P} / \mathrm{Q}$ channels. An alternative scenario, illustrated in Fig. $4 \mathrm{~b}$, can occur if the PF activity alone hyperpolarises the $V_{m}$ of the PN, for instance through MLI-mediated feed-forward inhibition or by activation of mGluR7s. Thus, the first CF input would activate $T$ channels while the second CF input, occurring when the $V_{m}$ of the $P N$ is restored to a standing depolarised state, would activate $\mathrm{P} / \mathrm{Q}$ channels. Notably, the $\mathrm{Ca}^{2+}$ transient associated with the second CF input would be amplified by the mGluR1-mediated saturation of the ECB, 
regardless of whether the $\mathrm{Ca}^{2+}$ influx is mediated by the T-type or the P/Q-type VGCC. Interestingly, these hypotheses are consistent with the idea of "intrinsic" learning and memory in the cerebellum recently proposed in two perspective articles $[99,100]$. While these hypotheses are both, at this stage, only pure speculations, the careful analysis of the $V_{m}$ should be included in all future investigations of synaptic plasticity induction protocols.

\section{Perspectives}

To answer the general question formulated in the title of this review, three specific questions must be addressed by future experimental investigations: 1 . What can cause activity dependent hyperpolarisation? 2. When does hyperpolarisation occur in vivo? 3. How does hyperpolarisation correlate with induction of synaptic plasticity? While activation of $\mathrm{K}^{+}$channels can be in principle still investigated in brain slices, these questions must be addressed by measuring dendritic $\mathrm{V}_{\mathrm{m}}$ in vivo. Interestingly, Roome and Kuhn [101] achieved simultaneous dendritic $\mathrm{V}_{\mathrm{m}}$ and $\mathrm{Ca}^{2+}$ imaging using an organic voltage indicator, combined with somatic electrical recording from spontaneously active PNs in awake mice. The preliminary results reported in this study suggest that, at a fine time scale, the spatiotemporal processing in PN dendrites is characterised by a high degree of complexity. The most promising approach is the one proposed by Villette et al. based on genetically encoded indicators [102], permitting to record dendritic activity for long periods and to associate these recordings with protocols of cerebellar learning. In summary, the recent findings on the critical role in $\mathrm{V}_{\mathrm{m}}$ in selecting $\mathrm{Ca}^{2+}$ sources associated with a CF input pave the way for future investigations using the information on $V_{m}$ as a possible key for cerebellar synaptic plasticity. 


\section{References}

[1] Eccles JC, Ito M, Szentagotai J. The cerebellum as a neuronal machine. Berlin, Heidelberg, New York: Springer Verlag 1967.

[2] Jelitai M, Puggioni P, Ishikawa T, Rinaldi A, Duguid I. Dendritic excitation-inhibition balance shapes cerebellar output during motor behaviour. Nat Commun. 2016; 7: 13722.

[3] Marr D. A theory of cerebellar cortex. J Physiol. 1969; 202: 437-70.

[4] Albus JS. A theory of cerebellar function. Math Biosci. 1971; 28: 167-71.

[5] Ito M, Sakurai M, Tongroach P. Climbing fiber induced depression of both mossy fiber responsiveness and glutamate sensitivity of cerebellar Purkinje cells. J Physiol. 1982; 324: 113-34.

[6] Suvrathan A, Raymond JL (2018) Depressed by Learning-Heterogeneity of the Plasticity Rules at Parallel Fiber Synapses onto Purkinje Cells. Cerebellum. 2018; 17: 747-55.

[7] Jörntell H, Hansel C. Synaptic memories upside down: bidirectional plasticity at cerebellar parallel fiber-Purkinje cell synapses. Neuron. 2006; 52: 227-38.

[8] Vogt KE, Canepari M. On the induction of postsynaptic granule cell-Purkinje neuron LTP and LTD. Cerebellum. 2010; 9: 284-90.

[9] Wang SS, Denk W, Hausser M. Coincidence detection in single dendritic spines mediated by calcium release. Nat Neurosci. 2000; 3: 1266-73.

[10] Brenowitz SD, Regehr WG. Associative short-term synaptic plasticity mediated by endocannabinoids. Neuron. 2005; 45: 419-31.

[11] Ait Ouares K, Canepari M. The Origin of Physiological Local mGluR1 Supralinear $\mathrm{Ca}_{2+}$ Signals in Cerebellar Purkinje Neurons. J Neurosci. 2020; 40: 1795-809.

[12] Napper RM, Harvey RJ. Number of parallel fibre synapses on an individual Purkinje cell in the cerebellum of the rat. J Comp Neurol. 1988; 274: 168-77.

[13] Silver RA, Momiyama A, Cull-Candy SG. Locus of frequency-dependent depression identified with multipleprobability fluctuation analysis at rat climbing fibre-Purkinje cell synapses. J Physiol. 1998; 510: 881-902.

[14] Knöpfel T, Vranesic I, Staub C, Gähwiler BH. Climbing Fibre Responses in Olivo-cerebellar Slice Cultures. II. Dynamics of Cytosolic Calcium in Purkinje Cells. Eur J Neurosci. 1991; 3:343-8.

[15] Miyakawa H, Lev-Ram V, Lasser-Ross, N, Ross WN. Calcium transients evoked by climbing fiber and parallel fiber synaptic inputs in guinea pig cerebellar Purkinje neurons. J Neurophysiol. 1992; 68:1178-89.

[16] Canepari M, Vogt KE. Dendritic spike saturation of endogenous calcium buffer and induction of postsynaptic cerebellar LTP. PLoS ONE. 2008; 3: e4011. 
[17] Ait Ouares K, Filipis L, Tzilivaki A, Poirazi P, Canepari M. Two Distinct Sets of $\mathrm{Ca}_{2+}$ and $\mathrm{K}_{+}$Channels Are Activated at Different Membrane Potentials by the Climbing Fiber Synaptic Potential in Purkinje Neuron Dendrites. J Neurosci. 2019; 39: 1969-81.

[18] Fierro L, Llano I. High endogenous calcium buffering in Purkinje cells from rat cerebellar slices. J Physiol. 1996; 496: 617-25.

[19] Bastianelli E. Distribution of calcium-binding proteins in the cerebellum. Cerebellum. 2003 2: 242-62.

[20] Schmidt H, Stiefel KM, Racay P, Schwaller B, Eilers J. Mutational analysis of dendritic Ca2+ kinetics in rodent Purkinje cells: role of parvalbumin and calbindin D28k. J Physiol. 2003; 551: 13-32.

[21] Maeda H, Ellis-Davies GC, Ito K, Miyashita, Y Kasai H. Supralinear Ca2+ signaling by cooperative and mobile Ca2+ buffering in Purkinje neurons. Neuron. 1999. 24: 989-1002.

[22] Otsu Y, Marcaggi P, Feltz A, Isope P, Kollo M, Nusser Z, et al. Activity-dependent gating of calcium spikes by Atype K+ channels controls climbing fiber signaling in Purkinje cell dendrites. Neuron. 2014; 84:137-51.

[23] Hildebrand ME, Isope P, Miyazaki T, Nakaya T, Garcia E, Feltz A, et al. Functional coupling between mGluR1 and Cav3.1 T-type calcium channels contributes to parallel fiber-induced fast calcium signaling within Purkinje cell dendritic spines. J Neurosci. 2009; 29: 9668-82.

[24] Isope P, Hildebrand ME, Snutch TP. Contributions of T-type voltage-gated calcium channels to postsynaptic calcium signaling within Purkinje neurons. Cerebellum. 2012; 11:651-65.

[25] Canepari M, Ogden D. Evidence for protein tyrosine phosphatase, tyrosine kinase, G-protein regulation of the parallel fiber metabotropic slow EPSC of rat cerebellar Purkinje neurons. J Neurosci. 2003; 23: 4066-71.

[26] Anwar H, Hong S, De Schutter E. Controlling Ca2+-activated K+ channels with models of Ca2+ buffering in Purkinje cells. Cerebellum. 2012; 11: 681-93.

[27] Canepari M, Papageorgiou G, Corrie JET, Watkins C, Ogden D. The conductance underlying the parallel fibre slow EPSP in rat cerebellar Purkinje neurones studied with photolytic release of I-glutamate. J Physiol. 2001; 533: $765-72$.

[28] Canepari M, Auger C, Ogden D. Ca2+ ion permeability and single-channel properties of the metabotropic slow EPSC of rat Purkinje neurons. J Neurosci. 2004; 24: 3563-73.

[29] Hartmann J, Dragicevic E, Adelsberger H, Henning HA, Sumser M, Abramowitz J, et al. TRPC3 channels are required for synaptic transmission and motor coordination. Neuron. 2008; 59: 392-8.

[30] Ady V, Perroy J, Tricoire L, Piochon C, Dadak S, Chen X, et al. Type 1 metabotropic glutamate receptors (mGlu1) trigger the gating of GluD2 delta glutamate receptors. EMBO Rep. 2014; 15: 103-9.

[31] Schmidt H, Eilers J. Spine neck geometry determines spino-dendritic cross-talk in the presence of mobile endogenous calcium binding proteins. J Comput Neurosci. 2009; 27: 229-43. 
[32] Schmidt H, Kunerth S, Wilms C, Strotmann R, Eilers J. Spino-dendritic cross-talk in rodent Purkinje neurons mediated by endogenous Ca2+-binding proteins. J Physiol. 2007; 581: 619-29.

[33] Loewenstein Y, Mahon S, Chadderton P, Kitamura K, Sompolinsky H, Yarom Y, Häusser M. Bistability of cerebellar Purkinje cells modulated by sensory stimulation. Nat Neurosci. 2005; 8: 202-11.

[34] Kitamura K. Judkewitz B. Kano M. Denk W. Häusser M. Targeted patch-clamp recordings and single-cell electroporation of unlabeled neurons in vivo. Nat. Methods. 2008; 5: 61-67.

[35] Arlt C, Häusser M. Microcircuit Rules Governing Impact of Single Interneurons on Purkinje Cell Output In Vivo. Cell Rep. 2020; 30: 3020-35.

[36] Williams SR, Christensen SR, Stuart GJ, Häusser M. Membrane potential bistability is controlled by the hyperpolarization- activated current $\mathrm{I}(\mathrm{H})$ in rat cerebellar Purkinje neurons in vitro. J Physiol. 2002; 539: 46983.

[37] Yuen GL, Hockberger PE, Houk JC. Bistability in cerebellar Purkinje cell dendrites modelled with high- threshold calcium and delayed-rectifier potassium channels. Biol Cybern. 1995; 73: 375-88.

[38] Arancillo M, White JJ, Lin T, Stay TL, Sillitoe RV. In vivo analysis of Purkinje cell firing properties during postnatal mouse development. J Neurophysiol. 2015; 113: 578-91.

[39] Zhou H, Lin Z, Voges K, Ju C, Gao Z, Bosman LW, et al. Cerebellar modules operate at different frequencies. Elife. 2014; 3: e02536.

[40] Wu B, Blot FG, Wong AB, Osório C, Adolfs $\mathrm{Y}$, Pasterkamp RJ, et al. TRPC3 is a major contributor to functional heterogeneity of cerebellar Purkinje cells. Elife. 2019; 8: e45590.

[41] Rancz EA, Häusser M. Dendritic calcium spikes are tunable triggers of cannabinoid release and short-term synaptic plasticity in cerebellar Purkinje neurons. J Neurosci. 2006; 26: 5428-37.

[42] Wulff $P$, Schonewille M, Renzi M, Viltono L, Sassoè-Pognetto M, Badura A, et al. Synaptic inhibition of Purkinje cells mediates consolidation of vestibulo-cerebellar motor learning. Nat Neurosci. 2009; 12: 1042-9.

[43] Blazquez PM, Yakusheva TA. GABA-A Inhibition Shapes the Spatial and Temporal Response Properties of Purkinje Cells in the Macaque Cerebellum. Cell Rep. 2015; 11: 1043-53.

[44] Rowan MJM, Bonnan A, Zhang K, Amat SB, Kikuchi C, Taniguchi H, et al. Graded Control of Climbing-FiberMediated Plasticity and Learning by Inhibition in the Cerebellum. Neuron. 2018; 99: 999-1015.

[45] Gaffield MA, Rowan MJM, Amat SB, Hirai H, Christie JM. Inhibition gates supralinear $\mathrm{Ca}_{2+}$ signaling in Purkinje cell dendrites during practiced movements. Elife. 2018; 7: e36246.

[46] Kuo CC, Yang S. Recovery from inactivation of t-type ca2+ channels in rat thalamic neurons. J Neurosci. 2001; 21: 1884-92.

[47] Szapiro G, Barbour B. Multiple climbing fibers signal to molecular layer interneurons exclusively via glutamate spillover. Nat Neurosci. 2007; 10: 735-42. 
[48] Hosy E, Piochon C, Teuling E, Rinaldo L, Hansel C. SK2 channel expression and function in cerebellar Purkinje cells. J Physiol. 2011; 589: 3433-40.

[49] Cingolani LA, Gymnopoulos M, Boccaccio A, Stocker M, Pedarzani P. Developmental regulation of smallconductance Ca2+-activated K+ channel expression and function in rat Purkinje neurons. J Neurosci. 2002; 22: 4456-67.

[50] Luján R, Aguado C, Ciruela F, Arus XM, Martín-Belmonte A, Alfaro-Ruiz R, et al. SK2 Channels Associate With mGlu $_{1 \alpha}$ Receptors and Cav2.1 Channels in Purkinje Cells. Front Cell Neurosci. 2018; 12: 311.

[51] Titley HK, Watkins GV, Lin C, Weiss C, McCarthy M, Disterhoft JF, Hansel C. Intrinsic Excitability Increase in Cerebellar Purkinje Cells after Delay Eye-Blink Conditioning in Mice. J Neurosci. 2020; 40: 2038-46.

[52] Indriati DW, Kamasawa N, Matsui K, Meredith AL, Watanabe M, Shigemoto R. Quantitative localization of Cav2.1 (P/Q-type) voltage-dependent calcium channels in Purkinje cells: somatodendritic gradient and distinct somatic coclustering with calcium-activated potassium channels. J Neurosci. 2013; 33: 3668-78.

[53] Canepari M, Ogden D. Kinetic, pharmacological and activity-dependent separation of two Ca2+ signalling pathways mediated by type 1 metabotropic glutamate receptors in rat Purkinje neurones. J Physiol. 2006; 573: 65-82.

[54] Maeda N, Kawasaki T, Nakade S, Yokota N, Taguchi T, Kasai M, Mikoshiba K. Structural and functional characterization of inositol 1,4,5-trisphosphate receptor channel from mouse cerebellum. J Biol Chem. 1991; 266: 1109-16.

[55] Finch EA, Augustine GJ. Local calcium signalling by inositol-1,4,5-trisphosphate in Purkinje cell dendrites. Nature. 1998; 396: 753-6.

[56] Takechi H, Eilers J, Konnerth A. A new class of synaptic response involving calcium release in dendritic spines. Nature. 396: 757-60.

[57] Khodakhah K, Armstrong CM. Induction of long-term depression and rebound potentiation by inositol trisphosphate in cerebellar Purkinje neurons. Proc Natl Acad Sci U S A. 1997; 94: 14009-14.

[58] Inoue T, Kato K, Kohda K, Mikoshiba K Type 1 inositol 1,4,5-trisphosphate receptor is required for induction of long-term depression in cerebellar Purkinje neurons. J Neurosci. 1998; 18: 5366-73.

[59] Kano M, Garaschuk O, Verkhratsky A, Konnerth, A. Ryanodine receptor-mediated intracellular calcium release in rat cerebellar Purkinje neurones. J Physiol. 1995; 487: 1-16.

[60] Kohda K, Inoue T, Mikoshiba K. Ca2+ release from Ca2+ stores, particularly from ryanodine-sensitive Ca2+ stores, is required for the induction of LTD in cultured cerebellar Purkinje cells. J Neurophysiol. 1995; 74: 21848.

[61] Johansson F, Carlsson HA, Rasmussen A, Yeo CH, Hesslow G. Activation of a Temporal Memory in Purkinje Cells by the mGluR7 Receptor. Cell Rep. 2015; 13: 1741-6. 
[62] Niswender CM, Johnson KA, Luo Q, Ayala JE, Kim C, Conn PJ, Weaver CD. A novel assay of Gi/o-linked G protein-coupled receptor coupling to potassium channels provides new insights into the pharmacology of the group III metabotropic glutamate receptors. Mol Pharmacol. 2008; 73: 1213-24.

[63] Aguado C, Colón J, Ciruela F, Schlaudraff F, Cabañero MJ, Perry C, et al. Cell type-specific subunit composition of G protein-gated potassium channels in the cerebellum. J Neurochem. 2008; 105: 497-511.

[64] Tabata T, Haruki S, Nakayama H, Kano M. GABAergic activation of an inwardly rectifying K+ current in mouse cerebellar Purkinje cells. J Physiol. 2005; 563: 443-57.

[65] Konnerth A, Dreessen J, Augustine GJ. Brief dendritic calcium signals initiate long-lasting synaptic depression in cerebellar Purkinje cells. Proc Natl Acad Sci USA. 1992;89:7051-5.

[66] Karachot L, Kado RT, Ito M. Stimulus parameters for induction of long-term depression in in vitro rat Purkinje cells. Neurosci Res.1995; 21:161-8.

[67] Safo PK, Regehr WG. Endocannabinoids control the induction of cerebellar LTD. Neuron. 2005; 48: 647-59.

[68] Lev-Ram V, Wong ST, Storm DR, Tsien RY. A new form of cerebellar long-term potentiation is postsynaptic and depends on nitric oxide but not cAMP. Proc Natl Acad Sci USA. 2002;99: 8389-93.

[69] Coesmans M, Weber JT, De Zeeuw Cl, Hansel C. Bidirectional parallel fiber plasticity in the cerebellum under climbing fiber control. Neuron. 2004;18:691-700.

[70] Hartell NA. Strong activation of parallel fibers produces localized calcium transients and a form of LTD that spreads to distant synapses. Neuron. 1996;16:601-10.

[71] Bienenstock EL, Cooper LN, Munro PW. Theory for the development of neuron selectivity: orientation specificity and binocular interaction in visual cortex. J Neurosci. 1982; 2: 32-48.

[72] Conquet F, Bashir ZI, Davies CH, Daniel H, Ferraguti F, Bordi F, et al. Motor deficit and impairment of synaptic plasticity in mice lacking mGluR1. Nature. 1994;372:237-43.

[73] Aiba A, Kano M, Chen C, Stanton ME, Fox GD, Herrup K, et al. Deficient cerebellar long-term depression and impaired motor learning in mGluR1 mutant mice. Cell. 1994;79:377-88.

[74] Hartell NA. Induction of cerebellar long-term depression requires activation of glutamate metabotropic receptors. NeuroReport. 1994;5:913-6.

[75] Marcaggi P, Attwell D. Short- and long-term depression of rat cerebellar parallel fibre synaptic transmission mediated by synaptic crosstalk. J Physiol. 2007; 578: 545-50.

[76] Baude A, Nusser Z, Roberts JD, Mulvihill E, Mcllhinney RA, Somogyi P. The metabotropic glutamate receptor (mGluR1 alpha) is concentrated at perisynaptic membrane of neuronal subpopulations as detected by immunogold reaction. Neuron. 1993; 11: 771-87.

[77] Marcaggi P, Attwell D. Endocannabinoid signaling depends on the spatial pattern of synapse activation. Nat Neurosci. 2005; 8: 776-81. 
[78] Bower JM. The organization of cerebellar cortical circuitry revisited: implications for function. Ann N Y Acad Sci. 2002; 978: 135-55.

[79] Welsh JP, Yamaguchi H, Zeng XH, Kojo M, Nakada Y, Takagi A, et al. Normal motor learning during pharmacological prevention of Purkinje cell long-term depression. Proc Natl Acad Sci U S A. 2005;102:1716671.

[80] Schonewille M, Gao Z, Boele HJ, Veloz MF, Amerika WE, Simek AA, et al. Reevaluating the role of LTD in cerebellar motor learning. Neuron. 2011;70:43-50.

[81] Tanaka S, Kawaguchi SY, Shioi G, Hirano T. Long-term potentiation of inhibitory synaptic transmission onto cerebellar Purkinje neurons contributes to adaptation of vestibulo-ocular reflex. J Neurosci. 2013; 33: 17209-20.

[82] Ramakrishnan KB, Voges K, De Propris L, De Zeeuw Cl, D'Angelo E Tactile Stimulation Evokes Long-Lasting Potentiation of Purkinje Cell Discharge In Vivo. Front Cell Neurosci. 2016; 10:36.

[83] Salin PA, Malenka RC, Nicoll RA. Cyclic AMP mediates a presynaptic form of LTP at cerebellar parallel fiber synapses. Neuron. 1996; 16: 797-803.

[84] Qiu DL, Knöpfel T. An NMDA receptor/nitric oxide cascade in presynaptic parallel fiber-Purkinje neuron longterm potentiation. J Neurosci. 2007; 27: 3408-15.

[85] Casado M, Isope P, Ascher P. Involvement of presynaptic N-methyl-D-aspartate receptors in cerebellar longterm depression. Neuron. 2002; 33: 123-30.

[86] Bouvier G, Higgins D, Spolidoro M, Carrel D, Mathieu B, Léna C, et al. Burst-Dependent Bidirectional Plasticity in the Cerebellum Is Driven by Presynaptic NMDA Receptors. Cell Rep. 2016; 15:104-116.

[87] Ogasawara H, Doi T, Doya K, Kawato M. Nitric oxide regulates input specificity of long-term depression and context dependence of cerebellar learning. PLoS Comput Biol. 2007; 3:e179.

[88] Carey MR, Myoga MH, McDaniels KR, Marsicano G, Lutz B, Mackie K, Regehr WG. Presynaptic CB1 receptors regulate synaptic plasticity at cerebellar parallel fiber synapses. J Neurophysiol. 2011; 105: 958-63.

[89] Kreitzer AC, Regehr WG. Retrograde inhibition of presynaptic calcium influx by endogenous cannabinoids at excitatory synapses onto Purkinje cells. Neuron. 2001; 29: 717-27.

[90] Maejima T, Hashimoto K, Yoshida T, Aiba A, Kano M. Presynaptic inhibition caused by retrograde signal from metabotropic glutamate to cannabinoid receptors. Neuron. 2001; 31: 463-75.

[91] Wang DJ, Su LD, Wang YN, Yang D, Sun CL, Zhou L, et al. Long-term potentiation at cerebellar parallel fiberPurkinje cell synapses requires presynaptic and postsynaptic signaling cascades. J Neurosci. 2014; 34: 235564.

[92] Suvrathan A, Payne HL, Raymond JL. Timing Rules for Synaptic Plasticity Matched to Behavioral Function. Neuron. 2016; 92: 959-67. 
[93] Hirano T. Regulation and Interaction of Multiple Types of Synaptic Plasticity in a Purkinje Neuron and Their Contribution to Motor Learning. Cerebellum. 2018; 17: 756-65.

[94] Zang Y, De Schutter E. Climbing Fibers Provide Graded Error Signals in Cerebellar Learning. Front Syst Neurosci. 2019; 13: 46.

[95] Dean P, Porrill J, Stone JV. Visual awareness and the cerebellum: possible role of decorrelation control. Prog Brain Res. 2004; 144: 61-75.

[96] Dean P, Porrill J, Stone JV. Decorrelation control by the cerebellum achieves oculomotor plant compensation in simulated vestibulo-ocular reflex. Proc Biol Sci. 2002; 269: 1895-904.

[97] Bouvier G, Aljadeff J, Clopath C, Bimbard C, Ranft J, Blot A, et al. Cerebellar learning using perturbations. Elife. 2018; 7: e31599.

[98] Titley HK, Kislin M, Simmons DH, Wang SS, Hansel C. Complex spike clusters and false-positive rejection in a cerebellar supervised learning rule. J Physiol. 2019; 597: 4387-406.

[99] Titley HK, Brunel N, Hansel C. Toward a Neurocentric View of Learning. Neuron. 2017; 95: 19-32.

[100] Johansson F. Intrinsic memory of temporal intervals in cerebellar Purkinje cells. Neurobiol Learn Mem. 2019; 166: 107103.

[101] Roome CG, Kuhn B. Simultaneous dendritic voltage and calcium imaging and somatic recording from Purkinje neurons in awake mice. Nat Commun. 2018; 9: 3388.

[102] Villette V, Chavarha M, Dimov IK, Bradley J, Pradhan L, Mathieu B, et al. Ultrafast Two-Photon Imaging of a High-Gain Voltage Indicator in Awake Behaving Mice. Cell. 2019; 179: 1590-608. 

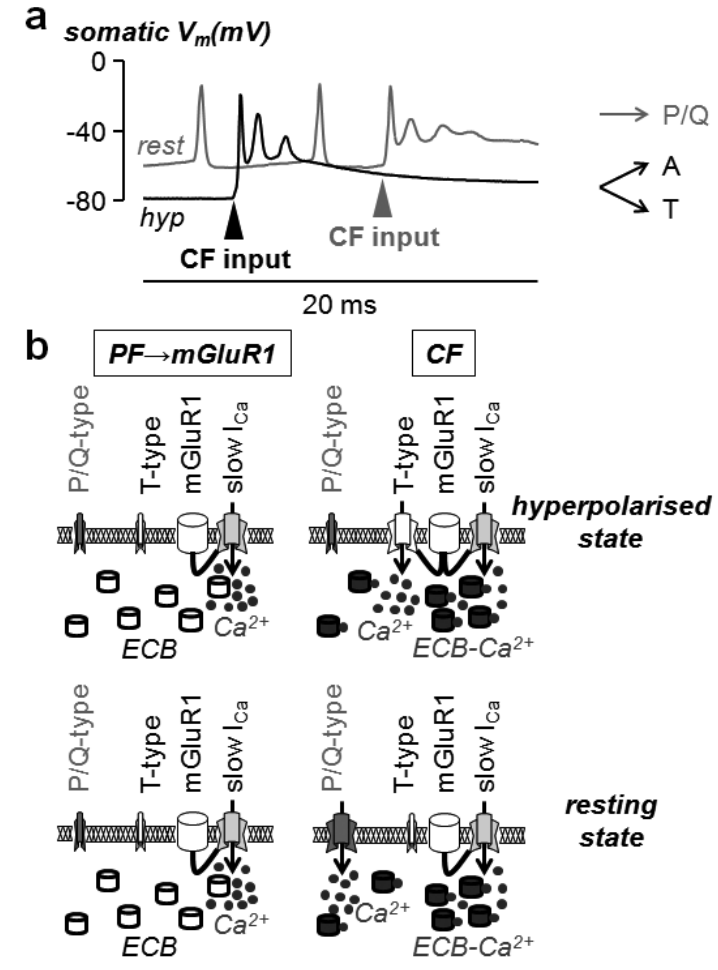

Fig. 1. $\mathrm{Ca}^{2+}$ sources responsible for $\mathrm{Ca}^{2+}$ transients associated with CF-EPSPs and mechanisms underlying supralinear amplification. a Example of CF Somatic $V_{m}$ associated with $C F$ inputs at initial hyperpolarised $V_{m}$ (black trace), activating $T$ and $A$ channels, and at resting $V_{m}$ (gray trace), activating $P / Q$ channels. $\mathbf{b}$ At both states, the slow $\mathrm{Ca}^{2+}$ current $\left(\mathrm{I}_{\mathrm{Ca}}\right)$ gated by mGluR1 activation following PF activity saturates the ECB amplifying the $\mathrm{CF} \mathrm{Ca}^{2+}$ transient. At the hyperpolarised state (top), this mechanism combines with the co-localised mGluR1-dependent potentiation of T-type VGCCs activated by the CF. At the resting state (bottom), this mechanism combines with the boosted CF activation of P/Q-type VGCCs, but the two mechanisms are not co-localised.

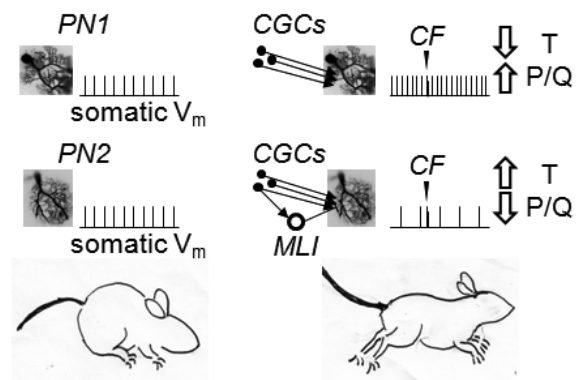

Fig. 2. Hypothesis on the role of feed-forward inhibition in determining the $\mathrm{Ca}^{2+}$ source associated with the CF input. On the left, two PNs (PN1 and PN2) firing at the same frequency when the mouse is at rest; on the right, PN1 increases the firing frequency with movement following excitation provided by CGC inputs while PN2 decreases the firing frequency following a dominance of MLI feed-forward inhibition; PN1 would favour activation of P/Q channels following a CF input while PN2 would favour activation of T channels following a CF input. 


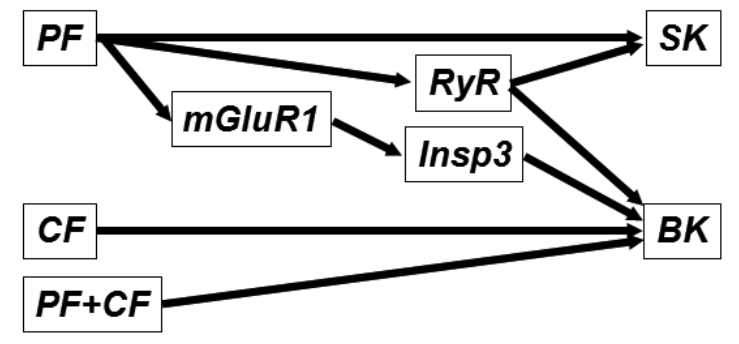

\section{b}

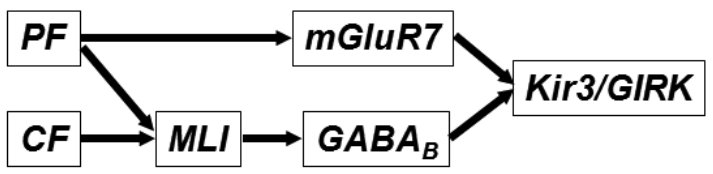

Fig. 3. Possible activity dependent pathways activating $\mathrm{K}^{+}$channels and producing hyperpolarising episodes. a Possible pathways leading to activation of $\mathrm{Ca}^{2+}$-activated $\mathrm{K}^{+}$channels. PF activity can activate $\mathrm{SK}$ channels either by directly inducing $\mathrm{Ca}^{2+}$ influx or through ryanodine receptors (RyR). PF activity can activate BK channels through RyR activation or through activation of mGluR1s that trigger $\mathrm{Ca}^{2+}$ release from stores. BK channels can be also activated by $\mathrm{Ca}^{2+}$ influx induced by the $\mathrm{CF}$ input either alone or paired with PF activity (PF+CF). b Possible pathways leading to activation of Kir3/GIRK $\mathrm{K}^{+}$channels. PF activity can activate mGluR7s that activate Kir3/GIRK channels. PF activity or CF inputs (via glutamate spillover) can activate MLIs that activate $\mathrm{GABA}_{B}$ receptors leading to activation of Kir3/GIRK channels.

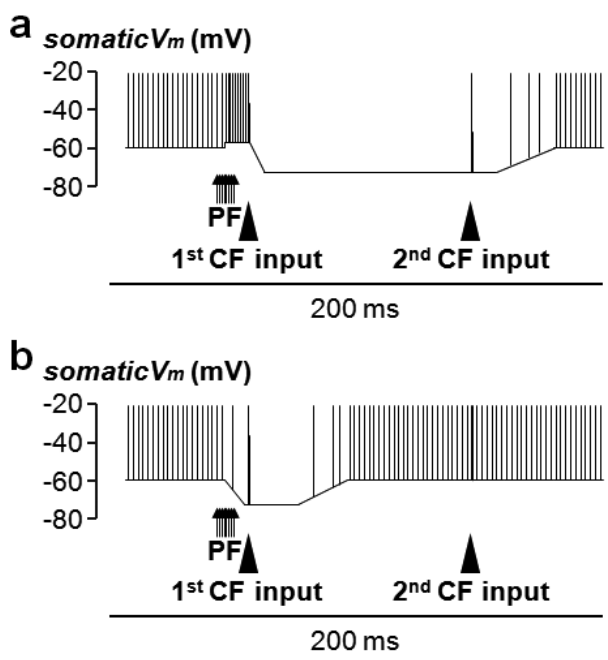

Fig. 4. Possible scenarios of plasticity induction protocols based on two consecutive CF inputs. a PF activity further depolarises $P N V_{m}$ and the first $C F$ input activates $P / Q$ channels leading to an episode of hyperpolarisation (for instance by activating $\mathrm{Ca}^{2+}$-activated $\mathrm{K}^{+}$channels); the second $\mathrm{CF}$ input occurring when $\mathrm{PN} \mathrm{V}_{\mathrm{m}}$ is hyperpolarised activates $T$ channels. b PF activity hyperpolarises PN $V_{m}$ (for instance by MLI-mediated feed-forward inhibition or by mGluR7s) and the first CF input activates $T$ channels; the second CF input occurring when $P N V_{m}$ is restored to a depolarised state $\mathrm{P} / \mathrm{Q}$ channels. In both cases the $\mathrm{Ca}^{2+}$ transient associated with the second $\mathrm{CF}$ input is locally amplified by mGluR1-mediated ECB saturation, but the sequence of the two $\mathrm{Ca}^{2+}$ signals is different for the first and second CF input. 